\title{
A REDUCTION THEOREM FOR THE ZARISKI MULTIPLICITY CONJECTURE
}

\author{
DAVID B. MASSEY
}

(Communicated by Haynes R. Miller)

\begin{abstract}
We prove that the Zariski multiplicity conjecture for families of hypersurfaces of dimension $\neq 2$ with isolated singularities is equivalent to the conjecture for families of hypersurfaces with line singularities.
\end{abstract}

Zariski has conjectured that multiplicity is an ambient topological invariant for (reduced) complex hupersurfaces [Z]. The truth of this conjecture is not known even for families of hypersurfaces with isolated singularities, where it is equivalent to: if $f_{t}:\left(\mathbb{C}^{n+1}, \underline{0}\right) \rightarrow(\mathbb{C}, 0)$ is a $\mu$-constant family (i.e., the Milnor number of $f_{t}$ is independent of $\left.t\right)$, then the multiplicity of $f_{t}$ at $\underline{0}:=\operatorname{mult}_{0}\left(f_{t}\right)$ is constant. (The conjecture is, however, known to be true for $\mu$-constant families when $f_{0}$ is a quasihomogeneous polynomial. See [G] and [O].) We shall show that the Zariski multiplicity conjecture is true for families of hypersurfaces with isolated singularities if and only if it is true for families of hypersurfaces with line singularities, ${ }^{1}$ provided $n \neq 2$.

Of course, it is not conceivable that the conjecture for line singularities is easier to prove than the conjecture for isolated singularities-however, it may be easier to look for counterexamples among families of line singularities.

We recall a result of Iomdine and Lê which appears in [I] and [L2] in the case of a complete intersection with a one-dimensional singular set. Here, we will only be concerned with the special case of a hypersurface with a line singularity (that is, we assume that the singular set is a coordinate axis). In what follows, if $\alpha$ is an ideal in a ring $A$, then $A_{f}$ will denote the ring obtained from $A$ by inverting $\left\{f^{n}\right\}_{n \geq 0}$ and $\alpha_{f}$ will denote the ideal generated by $\alpha$ in $A_{f}$.

Let $f:\left(\mathbb{C}^{n+1}, \underline{0}\right) \rightarrow(\mathbb{C}, 0)$ be a (reduced) polynomial in the variables $\left(x_{1}\right.$, $\left.\ldots, x_{n}, s\right)$ with the singular set of $f$ being precisely the $s$-axis, and suppose that $\left.f\right|_{V(s)}$ has an isolated singularity at the origin. Then, for $s_{0}$ small and

Received by the editors August 7, 1987 and, in revised form May 2, 1988 and June 26, 1988.

1980 Mathematics Subject Classification (1985 Revision). Primary 32B30, 14B05, 32C40. ities.

Key words and phrases. Zariski multiplicity conjecture, one-dimensional hypersurface singular-

${ }^{i}$ Throughout, by making an analytic coordinate change, we could replace the assumption of having a line singularity with the assumption of having a smooth one-dimensional singular set. 
nonzero, $f_{s_{0}}:=f_{\mid V\left(s-s_{0}\right)}$ has an isolated singularity at the origin and $\mu\left(f_{s_{0}}\right)$, the Milnor number of $f_{s_{0}}$, is independent of $s_{0}$; we denote this common value by $\stackrel{\circ}{\mu}(f)$. We also have in this situation that, for all k sufficiently large, $f+s^{\mathrm{k}}$ has an isolated singularity at the origin $\left(\left[\mathrm{L}_{2}\right],[\mathrm{M}]\right)$.

Now, let $\gamma_{f, s}$ denote the ideal $\left\langle\partial f / \partial x_{1}, \ldots, \partial f / \partial x_{n}\right\rangle_{f} \cap \mathscr{O}^{n+1}$ in $\mathscr{O}^{n+1}$. Then, as a germ at the origin, $V\left(\gamma_{f, s}\right)$ is the polar curve of $f$ with respect to the coordinate $s$ ([HL], $\left.\left[\mathrm{L}_{1}\right]\right)$.

Proposition (Iomdine-Lê formula [I], [L $\left.\left.\mathrm{L}_{2}\right]\right)$. Suppose $k>\operatorname{dim}_{\mathbb{C}}\left(\mathscr{O}_{\underline{0}}^{n+1} /\left(\gamma_{f, s}+\right.\right.$ $\left.\langle f\rangle) \mathscr{O}_{0}^{n+1}\right)$ and $f+s^{k}$ has an isolated singularity at the origin. Then,

$$
\mu\left(f+s^{k}\right)=b_{n}(f)-b_{n-1}(f)+k \stackrel{\circ}{\mu}(f),
$$

where $b_{i}(f)$ denotes the $i$ th Betti number of the Milnor fibre $f$ at the origin.

Remark. We shall need the bound which appears in the statement above. This bound does not appear explicitly in $\left[\mathrm{L}_{2}\right]$.

Of course, the Betti numbers of the Milnor fibre of $f$ are invariants of the ambient topological type of $V(f)$ at the origin (see $\left.\left[\mathrm{L}_{3}\right]\right)$. We would like to know the same thing about $\stackrel{\circ}{\mu}$.

Proposition. For hypersurfaces with smooth one-dimensional singular sets, ${ }^{2}$ $\stackrel{\circ}{\mu}(f)$ is an invariant of the ambient topological type of $V(f)$ at the origin.

Proof. By definition, for all $s_{0} \neq 0$ arbitrarily close to $0, \mu\left(f_{s_{0}}\right)=\stackrel{\circ}{\mu}$. Hence, for a fixed small $s_{0} \neq 0, f$ gives a $\mu$-constant family along the $s$-axis near $\left(\underline{0}, s_{0}\right)$ and thus the Milnor fibre of $f$ at $\left(\underline{0}, s_{0}\right)$ is a cross-product of a disc with the Milnor fibre of $f_{s_{0}}$ at $\left(\underline{0}, s_{0}\right)$ in $\mathbb{C}^{n} \times\left\{s_{0}\right\}$ ([L $\left.\left.\mathrm{L}_{1}\right]\right)$. In particular, $\stackrel{\circ}{\mu}$ can be recovered from the homotopy type of the Milnor fibre of $f$ at $\left(0, s_{0}\right)$ which is an invariant of the ambient topological type of $V(f)$ at $\left(\underline{0}, s_{0}\right)$ in $\mathbb{C}^{n+1}$. But, the topological type of $V(f)$ at points $\left(\underline{0}, s_{0}\right)$ arbitrarily close to the origin in $\mathbb{C}^{n+1}$ is determined by the topological type of $V(f)$ at the origin. (This is immediate since for $U$ and $U^{\prime}$ open neighborhoods of $\underline{0}$, if $h:(U, U \cap V(f), \underline{0}) \rightarrow\left(U^{\prime}, U^{\prime} \cap V(g), \underline{0}\right)$ is a homeomorphism, then for all $p \in U \cap V(f), h:(U, U \cap V(F), p) \rightarrow\left(U^{\prime}, U^{\prime} \cap V(g), h(p)\right)$ gives the ambient topological type at $p$.)

It is now easy to sketch the proof of the main result. One implication is trivial.

Suppose the multiplicity conjecture is true for families of line singularities. Let $f_{t}=f_{t}\left(x_{1}, \ldots, x_{n}\right)$ be a family of isolated origin singularities with constant ambient topological type (for $t$ near 0 ). Then, by considering $f_{t}$ as a family in one higher dimension, we have a family of line singularities with

\footnotetext{
2 It is, however, completely unclear that: if $f$ has a smooth one-dimensional singular set and $V(f)$ and $V(g)$ have the same ambient topological type at the origin, then $g$ has a smooth singular set.
} 
constant ambient topological type and so, by our assumption, mult ${ }_{\underline{0}}\left(f_{t}\right)$ must be constant.

Suppose, on the other hand, that the multiplicity conjecture is true for families of isolated singularities. Let $f_{t}=f_{t}\left(x_{1}, \ldots, x_{n}, s\right)$ be a (reduced) family of line singularities as above with constant ambient topological type (for $t$ near $0)$. Then, $b_{n}\left(f_{t}\right)-b_{n-1}\left(f_{t}\right)$ and $\stackrel{\circ}{\mu}\left(f_{t}\right)$ are constant in $t$. What we would like to do is use the Iomdine-Lê formula to conclude that: for $k$ sufficiently large, $f_{t}+s^{k}$ is a $\mu$-constant family of isolated singularities for all $t$ small.

Assuming this for the moment, we proceed. We must now make the additional assumption that $n \neq 2$. Then, as $\mu\left(f_{t}+s^{k}\right)$ is constant, the ambient topological type of $V\left(f_{t}+s^{k}\right)$ is constant by [LR] and so-since we are assuming the multiplicity conjecture for families of isolated singularities- mult $_{\underline{0}}\left(f_{t}+s^{k}\right)$ is constant. But, this is true for all $k$ sufficiently large; hence, $\operatorname{mult}_{\underline{0}}\left(f_{t}\right)$ must be constant.

The above proof will be complete once we prove the following uniform version of the Iomdine-Lê formula:

Proposition (Uniform Iomdine-Lê formula, [M]). Let $f_{t}=f_{t}\left(x_{1}, \ldots, x_{n}, s\right)$ be a family of line singularities as above. Then, for all $k$ sufficiently large,

$$
\mu\left(f_{t}+s^{k}\right)=b_{n}\left(f_{t}\right)-b_{n-1}\left(f_{t}\right)+k \stackrel{\circ}{\mu}\left(f_{t}\right)
$$

for all $t$ sufficiently small.

Proof. The proof is an exercise in commutative algebra.

First, if $k$ is large enough so that $f_{0}+s^{k}$ has an isolated singularity at $\underline{0}$, then $f_{t}+s^{k}$ must have an isolated singularity at $\underline{0}$ for all $t$ small.

Now, noting the bound given with the Iomdine-Lê formula, what we must show is that for $t_{0}$ small $\operatorname{dim}_{\mathbb{C}}\left(\mathscr{O}_{\underline{0}}^{n+1} /\left(\gamma_{f_{t_{0}}, s}+\left\langle f_{t_{0}}\right\rangle\right)\right)$ is a bounded function of $t_{0}$.

Let $A=\mathscr{O}_{\mathbb{C}^{n+1} \times \mathbb{C}}, \mathrm{m}_{t_{0}}=\left\langle\underline{x}, s, t-t_{0}\right\rangle$, and $\alpha=\left\langle\partial f / \partial x_{1}, \ldots, \partial f / \partial x_{n}\right\rangle$. As we are assuming that $f_{\left.0\right|_{1}(s)}$ has an isolated singularity at the origin, $\operatorname{dim}_{0} V(\alpha, s, t)$ $=0$ and thus we see that $V(\alpha) \subseteq \mathbb{C}^{n+2}$ is purely two-dimensional at $\underline{0}$. Letting $\lambda=\alpha A_{f} \cap A+f A$, we see that $V(\lambda)$ is the intersection of $V(f)$ with the union of the components of $V(\alpha)$ which are not contained in $V(f)$. Therefore, $V(\lambda)$ is purely one-dimensional at $\underline{0}$. Let $\beta$ denote the localization $\lambda_{\left\langle x_{1}, \ldots, x_{n}, s\right\rangle} \cap A$.

Note that $\left(\alpha+\left\langle t-t_{0}\right\rangle\right) A_{f} \cap A=\gamma_{f_{t_{0}}, s}$, and if $t_{0} \neq 0$ is small enough so that $V\left(x_{1}, \ldots, x_{n}, s\right)$ is the only component of $V(\lambda)$ containing $\left(\underline{0}, 0, t_{0}\right)$ then $\beta A_{\mathrm{m}_{t_{0}}}=\lambda A_{\mathrm{m}_{t_{0}}}$. Thus, for $t_{0} \neq 0$ small

$$
\begin{aligned}
(\beta+ & \left.\left\langle t-t_{0}\right\rangle\right) A_{\mathrm{m}_{t_{0}}}=\left(\lambda+\left\langle t-t_{0}\right\rangle\right) A_{\mathrm{m}_{t_{0}}} \\
& =\left(\alpha A_{f} \cap A+f A+\left\langle t-t_{0}\right\rangle\right) A_{\mathrm{m}_{t_{0}}} \subseteq\left(\left(\alpha+\left\langle t-t_{0}\right\rangle\right) A_{f} \cap A+f A\right) A_{\mathrm{m}_{t_{0}}} \\
& =\left(\gamma_{f_{t_{0}}, s}+f A\right) A_{\mathrm{m}_{t_{0}}},
\end{aligned}
$$


and hence

$$
\operatorname{dim}_{\mathbb{C}} \frac{\mathscr{O}_{0}^{n+1}}{\gamma_{f_{t_{0}}, s+\left\langle f_{t_{0}}\right\rangle}} \leq \operatorname{dim}_{\mathbb{C}} \frac{A_{\mathrm{m}_{t_{0}}}}{\left(\beta+\left\langle t-t_{0}\right\rangle\right) A_{\mathrm{m}_{t_{0}}}}=\operatorname{dim}_{\mathbb{C}} \frac{A}{\beta+\left\langle t-t_{0}\right\rangle}
$$

as $A /\left(\beta+\left\langle t-t_{0}\right\rangle\right)$ is already $\mathrm{m}_{t_{0}}$-local.

Finally, $\operatorname{dim}_{\mathrm{C}}\left(A /\left(\beta+\left\langle t-t_{0}\right\rangle\right)\right)$ is well known to be an upper-semicontinuous function of $t_{0}$ (by an application of Nakayama's lemma to the finitely generated $\mathbb{C}[t]$-module $A / \beta)$.

Note. There is an even more trivial proof of the above result, if one is willing to use some basic results on intersection numbers.

With this proposition proven, our earlier argument proves:

Theorem. The Zariski multiplicity conjecture is true for families of hypersurfaces of dimension $\neq 2$ with isolated singularities if and only if it is true for families of hypersurfaces with line singularities.

Remarks. One might wonder if such a result is also correct for families of hypersurfaces with arbitrary one-dimensional singular sets. The Iomdine-Lê formula (and the uniform version) are still obtainable in this case with $\stackrel{\circ}{\mu}$ replaced by $\sum_{\nu} n_{\nu} \stackrel{\circ}{\mu}_{\nu}$, where $\nu$ runs over all components of the singular set of $f, n_{\nu}$ is the degree of the restriction of the map $s$ to the curved $\nu$, and $\stackrel{\circ}{\mu}_{\nu}$ is the Milnor number of a generic hyperplane slice of $f$ at a point on $\nu$ sufficiently close to $\underline{0}$. The problem is to show that this number is an invariant of the ambient topological type of $V(f)$. As we already know that $\stackrel{\circ}{\mu}$ is such an invariant, it remains to be shown that $n_{\nu}$ is also an invariant (at least for generic $s$ ). So we have the following multiplicity problem:

Let $\nu$ be a (reduced) one-dimensional component of $\Sigma V(f)$ through the origin. Is mult $_{\underline{0}} \nu$ an invariant of the ambient topological type of $V(f)$ at the origin?

Aside from the generalization to arbitrary one-dimensional singular sets, one might also wonder about similar results for families of hypersurfaces with singular sets of higher dimension. The problem here is that no good analog of the Iomdine-Lê formula is known.

\section{REFERENCES}

[G] G. M. Greuel, Constant Milnor number implies constant multiplicity for quasihomogeneous singularities, Manuscripta Math., 56, Fasc. 2 (1986), 159-166.

[HL] H. Hamm and D. T. Lê, Un Theoreme de Zariski du type de Lefschetz, Ann. Sci. Ecole Norm. Sup. 6 (1973), 317-366.

[I] I. N. Iomdine, Complex varieties with singularities of dimension one, Sibirsk Mat. Zh. 15 (1974), 1061-1082.

[ $\left.\mathrm{L}_{1}\right]$ D. T. Lê, Calcul du nombre de cycle evanouisssant d'une hypersurface complexe, Ann. Inst. Fourier (Grenoble) 23 (1973), 261-270. 
$\left[\mathrm{L}_{2}\right]$ _ Ensembles analytiques complexes avec lieu singulier de dimension un (D'Apres I. N. Iomdine) Seminar on singularities (Paris, 1976/1977), Publ. Math. Univ. Paris VII 7 (1980), 87-95.

$\left[\mathrm{L}_{3}\right]$ _ Topologie des singuarlities des hypersurfaces complexes, Singularities a Cargese in Asterique 7 et 8 (1973), 171-182.

[LR] T. Lê and C. P. Ramanujam, The invariance of Milnor's number implies the invariance of the topological type, Amer. J. Math. 98 (1976), 67-78.

[M] D. B. Massey, Families of hypersurfaces with one-dimensional singular setes, Dissertation, Duke University, 1986.

[O] D. O'Shea, Topologically trivial deformations of isolated quasihomogeneous hypersurface singularities are equimultiple, Proc. Amer. Math. Soc. 100 (1987), 260-262.

[Z] O. Zariski, Open questions in the theory of singularities, Bull. Amer. Math. Soc. 77 (1971), 481-491.

Department of Mathematics, Northeastern University, Boston, Massachusetts, 02115 